\title{
Thermal Fatigue Life of Solder Bumps in $\mathrm{BGA}^{*}$
}

\author{
Minoru MUKAI**, Takashi KAWAKAMI**, \\ Kuniaki TAKAHASHI ${ }^{* * *}$, Kikuo KISHIMOTO**** \\ and Tosikaz SHIBUYA****
}

\begin{abstract}
Fatigue life estimation of solder bumps is one of the most critical technologies for the development of ball grid array packages (BGA). In this study, strain singularity was examined based on the analytical results for the primary solder bump model. The result suggested that it was desirable to employ the equivalent creep strain range occurring at a distance of $0.05 \mathrm{~mm}$ from the singularity point. The fatigue life estimation of the actual solder bumps was performed based on this consideration. The estimation result for the crack initiation was in good agreement with the experimental results. A simple estimation of the crack propagation based on the linear damage law was also proposed for convenience' sake. The trend of the simple estimation results corresponded well with the experimental results of crack propagation. Therefore, the fatigue life estimation of solder bumps based on the present method is satisfactory for engineering purposes.
\end{abstract}

Key Words: Thermal Stress, Fatigue, Life Prediction, Structural Analysis, Crack Propagation

\section{Introduction}

Surface mount technology has come into widespread use in the electronics industry. Devices such as LSI packages and chip components are soldered directly onto the surface of the printed circuit board $(\mathrm{PCB})$. Solder joints connect the devices mechanically as well as electrically to the PCB. Devices mounted on the $\mathrm{PCB}$ are subject to temperature variations arising as a result of the power switching and ambient temperature changes. Because of the differences in thermal expansion coefficients of the various materials on the $\mathrm{PCB}$, thermal stresses arise under thermal cycling. Since the solder material is

* Received 18th September, 1997

** Energy and Mechanical Research Laboratories, Research and Development Center, Toshiba Corporation, 4-1 Ukishima- ${ }^{-}$ho, Kawasaki-ku, Kawasaki 2100862, Japan

*** Ome Works, Toshiba Corporation, 2-9 Suehiro-cho, Ome-city, Tokyo 198-0025, Japan

**** Dept. of Mechanical and Intelligent Systems Eng., Tokyo Institute of Technology, 2-12-1 Ohokayama, Meguro-ku, Tokyo 152-8552, Japan mechanically the most ductile part, low cycle fatigue cracking may occur in the solder joints. Recent trends in LSI package development indicate a shift toward more solder joints and larger package sizes, and temperature changes under field conditions are also becoming greater. Consequently, fatigue life prediction of solder joints has become one of the most important research areas in the development of reliable electronic components. There have been, therefore, many studies of fatigue life estimation for solder joints ${ }^{(1),(2)}$.

In previous papers, the authors proposed an estimation method for fatigue life of $\mathrm{Sn63- \textrm {Pb } 3 7}$ solder joints using a simple elastic-creep model, which consists of a linear spring element and Norton's creep law, and the equivalent creep strain range $e^{(3)-(7)}$. In these papers, this method based on the elastic-creep model was applied to the solder joints of a quad flat package and a pin grid array package, and the results revealed the advantage of this method. Recently, ball grid array packages (BGA), as shown in Fig. 1, have been widely adopted. In the estimation of the fatigue life of the BGA solder bumps, owing to the crack initiation in the vicinity of the interface, it is indis- 


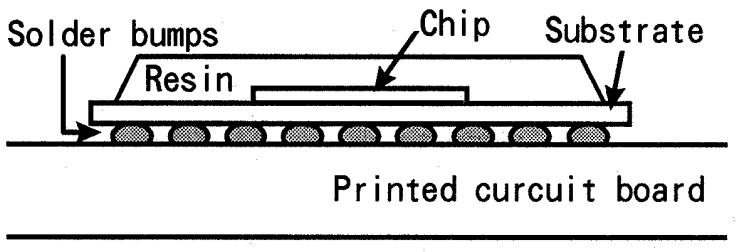

Fig. 1 BGA

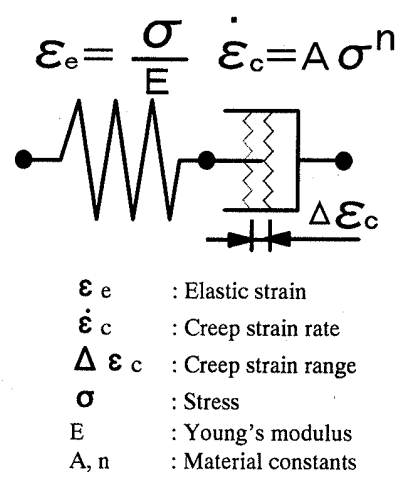

Fig. 2 Elastic ${ }^{-}$creep model

pensable to take account of the effects of stress-strain singularity. For this reason, the accurate fatigue life estimation of BGA is more complicated than that of other packages. In the present study, this method using the elastic ${ }^{-}$-reep model was applied to the solder bumps, based upon an assumption of an estimation point located at some distance from the singularity point. A simple estimation of crack propagation was also examined based on this assumption and linear damage law.

\section{Elastic-Creep Model of Sn63-Pb37}

Constructing a suitable material model of the Sn63-Pb37 solder is the most important issue in the stress analysis. Solder materials are alloys with low melting points, and their deformation behavior is nonlinear even at room temperature. In particular, their creep behavior, which is a time-dependent inelasticity, is significant. The authors have previously proposed a simple elastic-creep model to express the deformation behavior of $\mathrm{Sn} 63-\mathrm{Pb} 37$ solder, and also used this model in this study. This model is shown in Fig. 2, and the creep behavior is assumed to be expressed by Norton's law;

$$
\dot{\varepsilon}_{c}=A \sigma^{n}
$$

where $\dot{\varepsilon}_{c}$ is the equivalent creep strain rate, $\sigma$ is the equivalent stress, and $A$ and $n$ are material constants. The elastic and creep properties of Sn63-Pb37 solder at typical temperatures are listed in Table $1^{(3),(8)}$. Experimental results of $\mathrm{Sn} 63-\mathrm{Pb} 37$ solder materials have shown that an elastic-creep model is adequate for characterizing the creep behavior of materials in the low strain rate range. In this study, this elasticcreep model was employed for solder material in all
Table 1 Material properties of $\mathrm{Sn} 63-\mathrm{Pb} 37$ solder

\begin{tabular}{|c|c|c|c|}
\hline & $\begin{array}{c}\text { Young's } \\
\text { modulus } \\
(\mathrm{MPa})\end{array}$ & $\begin{array}{c}\text { Poisson's } \\
\text { ratio }\end{array}$ & $\begin{array}{c}\text { Thermal } \\
\text { expansion } \\
\text { coefficient } \\
\left(/{ }^{\circ} \mathrm{C}\right)\end{array}$ \\
\hline Solder & $2.00 \times 10^{4}$ & 0.39 & $2.47 \times 10^{-5}$ \\
\hline
\end{tabular}

\begin{tabular}{|c|c|c|}
\hline \multirow{2}{*}{$\begin{array}{c}\text { Temperature } \\
\left({ }^{\circ} \mathrm{C}\right)\end{array}$} & \multicolumn{2}{|c|}{$\boldsymbol{\varepsilon}_{\mathrm{c}}=\mathrm{A}^{\mathrm{n}}$} \\
\cline { 2 - 3 } & $\mathrm{A}(1 \mathrm{MPa} / \mathrm{hr})$ & $\mathrm{n}$ \\
\hline-65 & $6.545 \times 10^{-17}$ & 9.2 \\
\hline 0 & $3.570 \times 10^{-10}$ & 5.6 \\
\hline 25 & $1.975 \times 10^{-8}$ & 4.7 \\
\hline 50 & $6.417 \times 10^{-7}$ & 3.9 \\
\hline 100 & $7.044 \times 10^{-5}$ & 3.0 \\
\hline 150 & $2.089 \times 10^{-3}$ & 2.4 \\
\hline
\end{tabular}

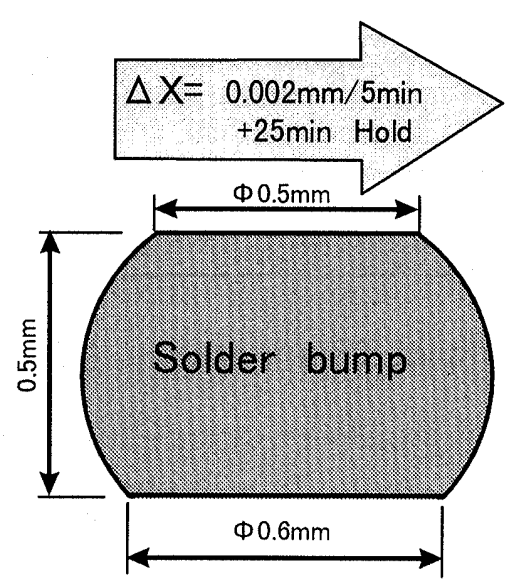

Fig. 3 Primary solder bump model

numerical analyses as follows.

\section{Strain Singularity of Solder Bumps}

In this study, first, strain singularities in the corner of solder bumps was examined based on the analytical results of a primary solder bump model under mechanical loads. The primary solder bump model is illustrated in Fig. 3. The relative displacement, $\Delta X$, which corresponds to the global mismatch of thermal expansion coefficients, was applied to the upper surface of the bump in the direction indicated by the arrow in Fig. 3 under the temperature condition of 25 degrees centigrade.

The distribution of the equivalent creep strain and the dependence on the distance from the surface in the direction of strain concentration are shown in Fig. 4. Because creep strain singularities occur in the corner of the solder bumps, a quantitative estimation of the number of cycles necessary to the fatigue based on the maximum value of the equivalent creep strain range is not significant. Since, in addition to various shapes of actual BGA bumps, they are subjected to 


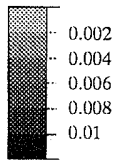

$\sum^{3}$

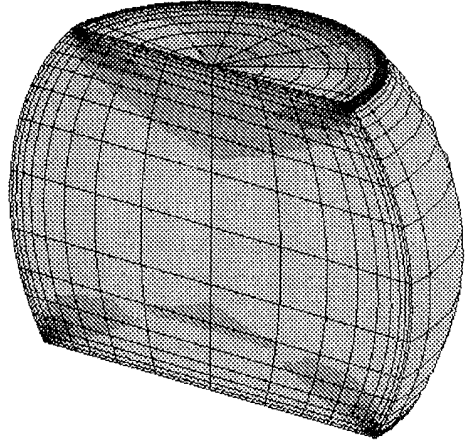

(a) Equivalent creep strain distribution

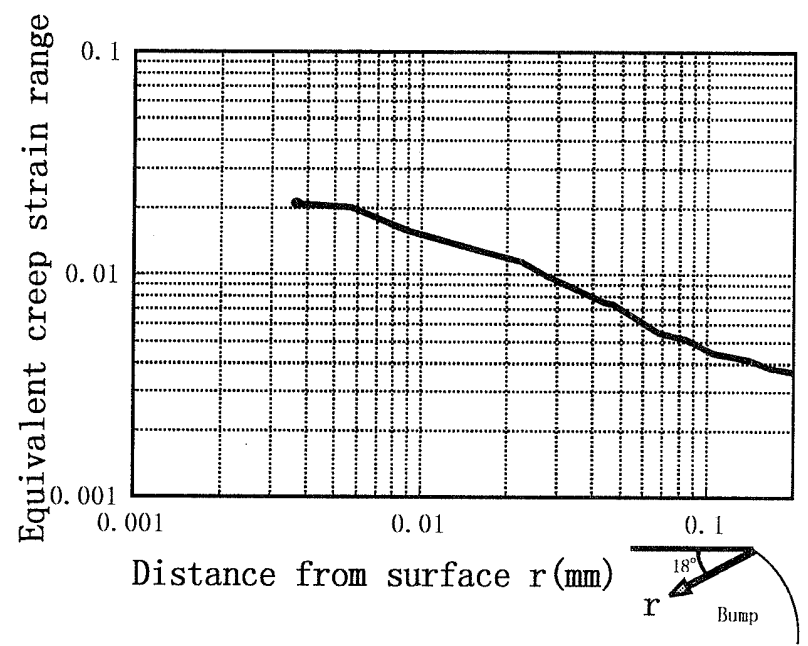

(b) Equivalent creep strain around the corner

Fig. 4 Equivalent creep strain in primary solder bump

mechanical and thermal loads, the trend of creep strain singularities may often be more complex, and can not be evaluated uniformly. In order to avoid the singularity problem of numerical analysis, it is of practical use to employ the equivalent creep strain range, which occurs at some distance from the singularity point. In consideration of the grain size of solder $(\sim 0.02 \mathrm{~mm})$, it has been assumed that an estimation point is located at a distance $0.05 \mathrm{~mm}$ from the singularity point on the surface of the bump. In this study, the same assumption was also applied to the strain singularity region in the crack tip. In the following chapter, this assumption is examined based on the results of the fatigue life estimation and thermal cycle test (TCT) of solder bumps in actual BGA.

\section{Thermal Fatigue Life of Solder Bumps in BGA}

Two stress analyses were performed in sequence to estimate the fatigue life of the solder bumps in a BGA. In the first stage, a global deformation analysis was carried out to obtain the displacement at the upper surface of the bump, and this result was used to assess the boundary condition for a detailed analysis. In the second stage, the individual solder bump, which
Table 2 Material properties

\begin{tabular}{|c|c|c|c|}
\hline & $\begin{array}{c}\text { Young's } \\
\text { modulus } \\
(\mathrm{MPa})\end{array}$ & $\begin{array}{c}\text { Poisson's } \\
\text { ratio }\end{array}$ & $\begin{array}{c}\text { Thermal } \\
\text { expansion } \\
\text { coefficient } \\
\left(/{ }^{\circ} \mathrm{C}\right)\end{array}$ \\
\hline Resin & $1.30 \times 10^{4}$ & 0.24 & $1.50 \times 10^{-5}$ \\
\hline Chip & $\begin{array}{c}\mathrm{E} 1=\mathrm{E} 2= \\
1.70 \times 10^{5} \\
\mathrm{E} 3=1.31 \times 10^{5}\end{array}$ & $\begin{array}{c}\cup 12=0.066 \\
\cup 31=0.364\end{array}$ & $3.5 \times 10^{-6}$ \\
\hline Substrate & $1.90 \times 10^{4}$ & 0.20 & $1.50 \times 10^{-5}$ \\
\hline $\begin{array}{c}\text { Printed } \\
\text { circuit } \\
\text { board }\end{array}$ & $1.80 \times 10^{4}$ & 0.20 & $1.60 \times 10^{-5}$ \\
\hline
\end{tabular}
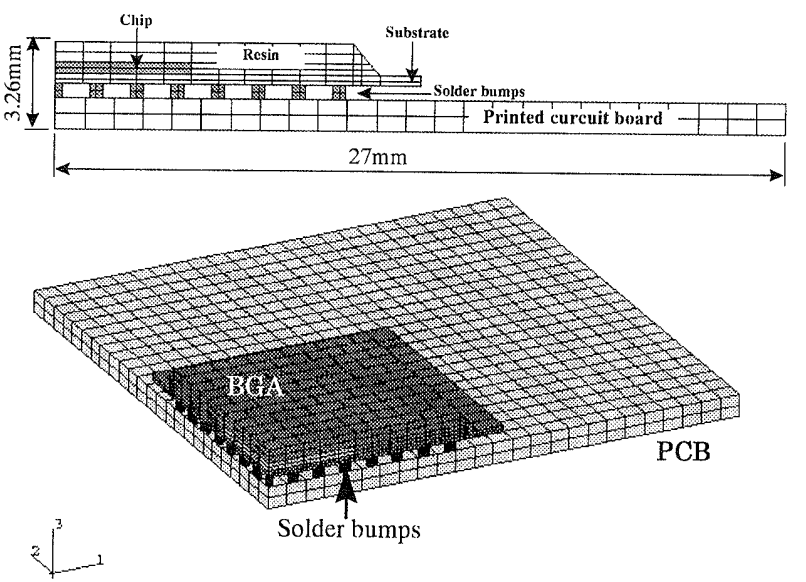

Fig. 5 Global model

is located in a region under a severe stress condition, was analyzed using the elastic-creep model of the solder material. The computation was carried out using the general purpose finite element structural analysis program ABAQUS (Version 5.4).

\section{1 Global deformation analysis}

Owing to the large number of solder bumps, it was difficult to perform an analysis of the global structure including the package, the $\mathrm{PCB}$, and each bump using a detailed analytical model. From an economic standpoint, analysis of only individual solder bumps is more reasonable for detailed analysis. This, however, requires knowledge of the relative displacement between the package and the PCB for use as the boundary condition. For this reason, the global deformation analysis was carried out to assess the boundary conditions at the upper surface of the bump. Due to the $1 / 4$ symmetry of this problem, the BGA, its bumps, and the PCB were modeled as shown in Fig. 5. The material properties of $\mathrm{BGA}$ and $\mathrm{PCB}$ used in this analysis are listed in Table 2, and solder bumps were analyzed using the elastic-creep model. The temperature history considered with the TCT condition is shown in Fig. 6, and includes temperature hold-times. 


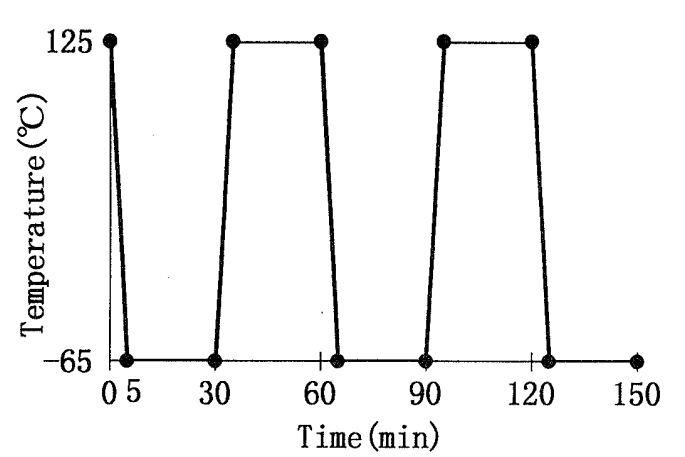

Fig. 6 Temperature history

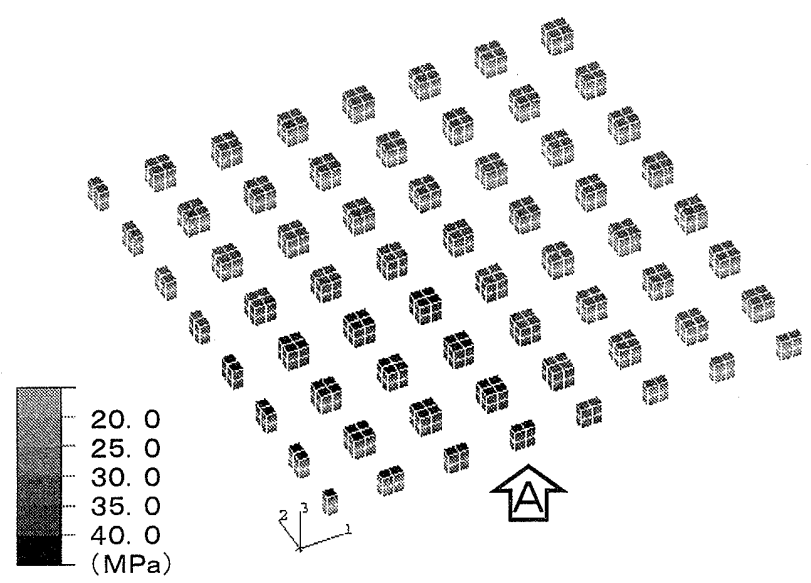

Fig. 7 Mises equivalent stress distributions in bumps of BGA

The distribution of the Mises equivalent stress in the solder region, shown in Fig. 7, was obtained after 5 minutes in the temperature history. Because of the high stiffness of silicon, the stress concentration area occurred in the edge of the chip, and this distribution trend corresponded well with the TCT results. In consideration of warps of the BGA and the PCB, a value of $0.0032 \mathrm{~mm}$ has been calculated for the relative displacement at the bump $\mathrm{A}$ in Fig. 7. In the present problem, this value was employed in the following analysis of the solder bump.

\section{2 Solder bump analyses}

The solder bump models of the BGA are illustrated in Fig. 8. A half section of the solder bump was considered due to the symmetry of the system. Quadratic brick elements were used over the whole region, with fine elements generated around the corner of the bump to obtain details of the stress-strain singularity. In addition to the model of the initial shape, models with cracks, which were $0.05 \mathrm{~mm}$ and $0.1 \mathrm{~mm}$ in depth, were also prepared. The relative displacement obtained by the global analysis was applied to the upper surface of the bump in the direction indicated by the arrow in Fig. 8.

\section{3 Estimation of crack initiation}

The distribution of the equivalent creep strain

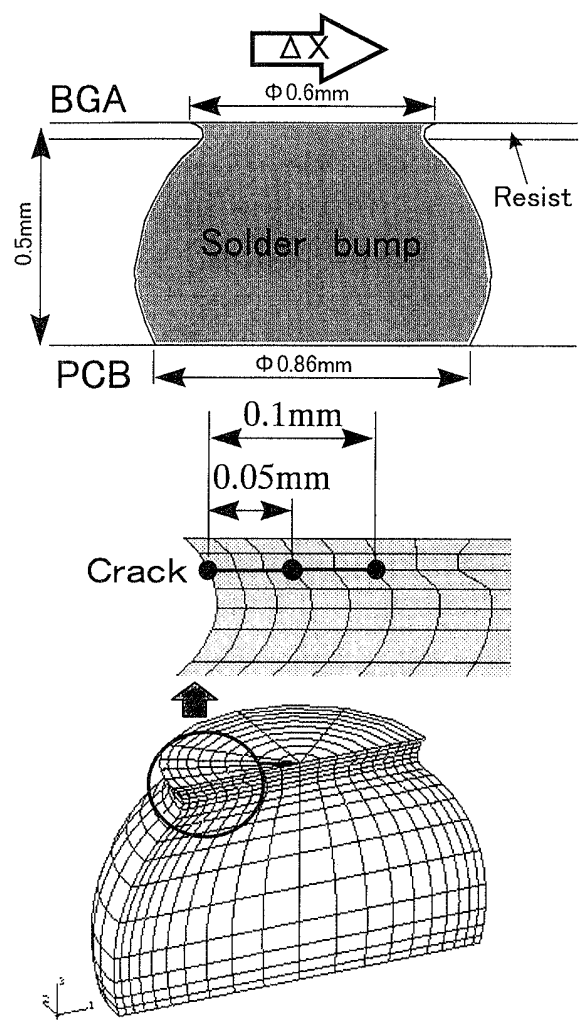

Fig. 8 Solder bump model of BGA
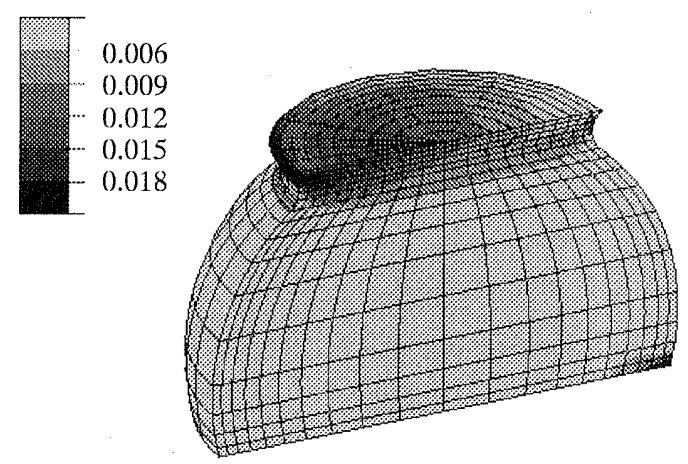

(a) Equivalent creep strain range distribution

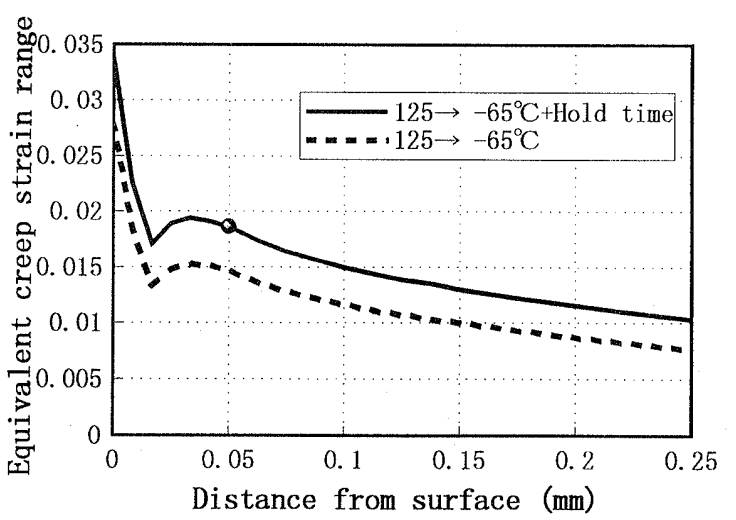

(b) Relationship between equivalent creep strain range and the distance from surface in the direction of the crack propagation

Fig. 9 Equivalent creep strain range in the model of the initial shape 
range in the model of the initial shape, and the details around the strain concentration region are shown in Fig. 9. Figure 10 shows a cross-sectional view of the solder bump after the actual TCT. It is clear that the strain concentration region coincides with the crack initiation site in the vicinity of the interface. The increase in creep strain during the hold-time occurs due to an elastic follow-up and stress redistribution phenomenon. Since the crack initiation site was located in the vicinity of the singularity point, values of the equivalent creep strain occurring at the surface were not reliable. Referring to Fig. 9, a value of 0.0186 has been obtained at a distance $0.05 \mathrm{~mm}$ from the surface of the bump.

The authors have previously carried out low cycle fatigue tests using thin-walled cylindrical specimens. The results showed that the effect of temperature and wave form on the Coffin-Manson plot was small. The following Coffin-Manson equation has been obtained to estimate thermal fatigue life $\mathrm{e}^{(7)}$ :

$$
N_{f}=f\left(\Delta \varepsilon_{i n}\right)=0.146\left(\Delta \varepsilon_{\text {in }}\right)^{-1.94}
$$

where $N_{f}$ is the number of cycles to failure, and $\Delta \varepsilon_{\text {in }}$ is the inelastic strain range. From a value of 0.0186 , the number of cycles to the crack initiation was estimated to be 330 cycles. Since macroscopic cracks with depth of about $0.04 \mathrm{~mm}$ were observed in the vicinity of the interface after 250 cycles of actual TCT, this estimation result is satisfactory.

\section{4 Simple estimation of crack propagation}

The estimation method of crack propagation based on the fracture mechanics is widely known, and it has been previously shown that the crack propagation at steady state of bulk solder material could be evaluated $^{(9)}$. However, unsteady crack propagation, which occurs immediately after crack initiation, is more significant than steady crack propagation, because the size of solder bumps is small. It is a little complex to apply the estimation method of steady crack propagation to micro solder bumps, because the method for taking account of the great fluctuation of previous damage is not clear. In addition, the applica-

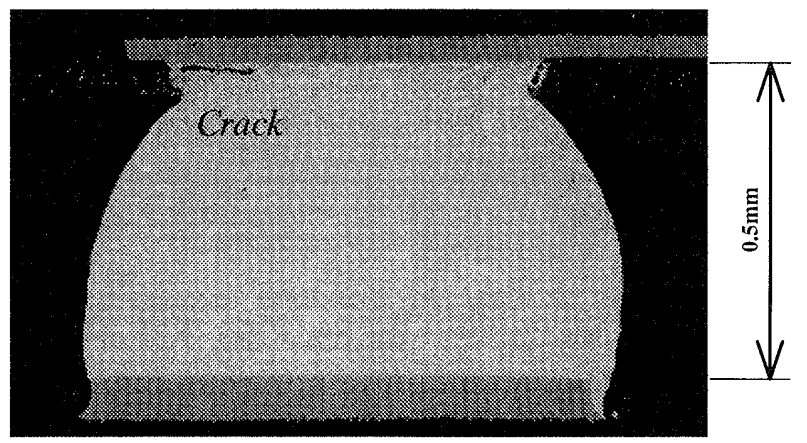

Fig. 10 Solder bump after TCT $\left(125^{\circ} \mathrm{C} \Leftrightarrow-65^{\circ} \mathrm{C}\right)$ bility of fracture mechanics to the crack propagation through the thermal stress field in micro structures such as the solder bumps has not also been investigated sufficiently. Therefore, in the case of the reliability design of the solder bumps, the construction of the estimation of crack propagation based on the fracture mechanics requires further investigation.

In this study a simple estimation of the crack propagation was carried out based on the linear damage law for the sake of convenience. The distributions of the equivalent creep strain range occurring in the strain concentration region, and the dependence on the distance from the surface are shown in Fig. 11. Since the region where high values of equivalent creep strain occurred was widespread in the direction of the crack propagation, previous damage, which arose before the crack initiation and arrival, cannot be neglected.

The simple estimation method presented is described as following. In the case of the model of a crack with a depth of $0.05 \mathrm{~mm}$, it was assumed that an estimation point is located at a distance $0.1 \mathrm{~mm}$ from the surface of the bump. A value of the equivalent creep strain range marked $\square$ on the curve $A$, which is the result of the model of the initial shape, in Fig. 11

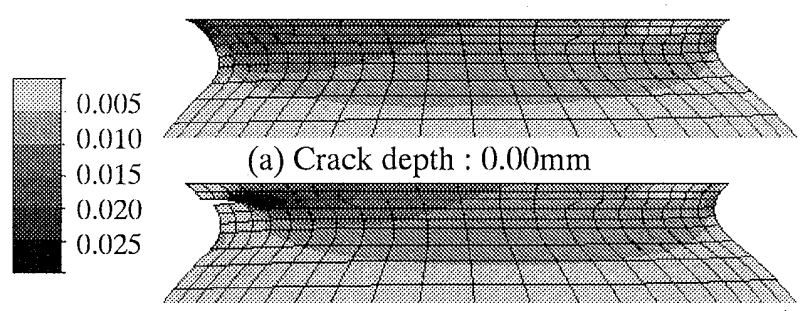

(b) Crack depth : $0.05 \mathrm{~mm}$

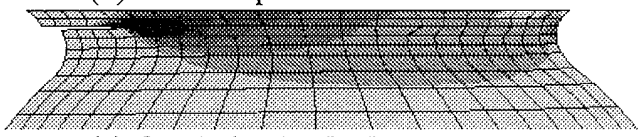

(c) Crack depth : $0.10 \mathrm{~mm}$

(a) Equivalent creep strain range distributions

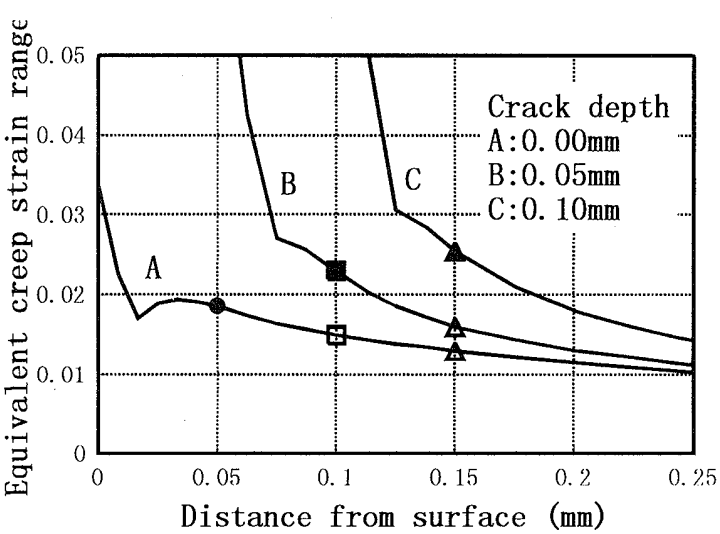

(b) Relationship between equivalent creep strain range and the distance from surface

Fig. 11 Equivalent creep strain range for each models 


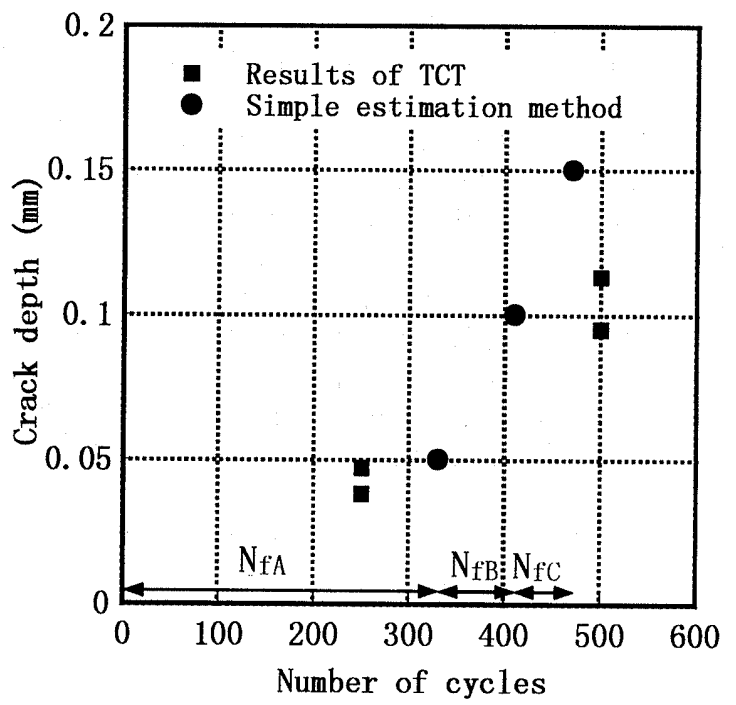

Fig. 12 Simple estimation of crack propagation in BGA solder bump

( $b$ ) is used to assess the previous damage at the estimation point. A cumulative damage value was calculated using this value of 0.015 and the number of 330 cycles obtained by the estimation of the crack initiation described in the previous paragraph. The number of cycles as a virgin material was obtained by substituting 0.023 as the equivalent creep strain range marked on the curve $B$, which is the result of the model with the crack $0.05 \mathrm{~mm}$ in depth, into the CoffinManson equation. The number of cycles necessary to the fatigue as a previously damaged material was determined by deducting the cumulative damage value based on the assumption of the linear damage law. The above procedure can be described by the following equation.

$$
N_{f B}=f(0.023) \times\left(1-N_{f A} / f(0.015)\right) \fallingdotseq 80
$$

where $N_{f A}(=f(0.0186))$ and $N_{f B}$ are the number of cycles to fatigue of the model of the initial shape and the model of the crack in depth of $0.05 \mathrm{~mm}$. The number of cycles to fatigue of the model for the crack with a depth of $0.1 \mathrm{~mm}, N_{f c}$, was also calculated by the same procedure.

$$
\begin{aligned}
& N_{f C}=f(0.0255) \times\left(1-N_{f A} / f(0.013)\right. \\
& \left.\quad-N_{f B} / f(0.016)\right) \doteqdot 60
\end{aligned}
$$

The relationship between the depth of the crack and the number of cycles is shown in Fig. 12 so as to compare the simple estimation results with the results of the TCT. The results of the simple estimation proposed correspond well with those of the TCT, in consideration of the large scattering of crack propagation behavior.

\section{Conclusions}

The strain singularity in the corner of solder bumps was examined based on the analytical results.
It was shown that the use of the equivalent creep strain range, which occurs at some distance from the singularity point, is of practical use in the design. In consideration of the grain size of solder, it has been assumed that an estimation point of the equivalent creep strain range is located at a distance $0.05 \mathrm{~mm}$ from the singularity point on the surface of the bump. This assumption was examined based on the results of the fatigue life estimation and thermal cycle test of solder bumps in actual BGA package. As a result, the estimated value for the number of cycles necessary to the crack initiation corresponded well with the experimental results. Consequently, the estimation of crack initiation using the equivalent creep strain range occurring at the above point is satisfactory for the reliability design of $\mathrm{BGA}$ solder bumps. When fracture mechanics is applied for the evaluation of the crack propagation in solder bumps, the applicability to the crack propagation at unsteady state is one important problem requiring future investigations. A simple estimation of the crack propagation based on the linear damage law was proposed for convenience' sake, and applied to the solder bumps. The trend of the simple estimation results for crack propagation corresponded well with the results of the TCT. Therefore, in the range of the engineering purposes, it was shown that the simple estimation method may be satisfactory for making a rough estimation of the crack propagation behavior in solder bumps.

\section{Acknowledgments}

The authors would like to thank Dr. Osamu Horigami and Mr. Setuo Yamamoto of the Toshiba R \& D Center for supporting this study. Thanks also to Mr. Hiroyuki Takahashi of the Toshiba R\&D Center for proffering the results of the crack propagation tests for Sn63-Pb37 solder.

\section{References}

( 1 Lau, J.H., Rice, D.W. and Avery, P.A., Elastoplastic Analysis of Surface-Mount Solder Joints, IEEE Trans. CHMT, Vol. 10, No. 3 (1987), p. 346357.

(2) Kitano, M., Kumazawa, T. and Kawai, S., A New Evaluation Method for Thermal Fatigue of Solder Joint, in Advances in Electronic Packaging, Proceedings of the 1992 Joint ASME/JSME Conference on Electronic Packaging, EEP-Vol. 1-1 (1992), p. 301-308.

(3) Mukai, M., Kawakami, T., Endo, T. and Takahashi, K., Elastic-Creep Behavior and Fatigue Life in an IC Package Solder Joint, Proceedings of JSME The 4th Computational Mechanics Conference, (in Japanese), No. 910-79 (1991), p. 223-224. 
(4) Mukai, M., Kawakami, T., Endo, T. and Takahashi, K., The Demonstration on Elastic-Creep Thermal Stress Analyses for IC's Sn63-Pb37 Solder Joints, Proceedings of JSME The 70th JSME Fall Annual Meeting, (in Japanese), No.920-78 (1992), p. 360-362.

( 5 ) Mukai, M., Kawakami, T., Endo, T., Hiruta, Y. and Takahashi, K., Elastic-Creep Thermal Stress Analyses for the SMT-PGA Package's Solder Joint, Proceedings of the ASMEWAM, AMD-Vol. 187 (1994), p. 197-203.

(6) Hiruta, Y., Hirano, N., Yamaji, Y., Mukai, M., Motoyama, Y., Homma, R., Ohno, J. and Sudo, T., An 820 Pin PGA for Ultralarge-Scale BiCMOS Devices, IEEE Trans. CHMT, Vol. 16, No. 8
(1993), p. 893-901.

(7) Mukai, M., Kawakami, T., Takahashi, K., Kishimoto, K. and Shibuya, T., Effects of HoldTime on Thermal Fatigue Life of Solder Joints, Trans. Jpn. Soc. Mech. Eng., (in Japanese), Vol. 63, No.611, A (1997), p. 1594-1600.

(8) Yebisuya, T. and Kawakubo, T., Creep and Tensile Properties of Cast $\mathrm{Bi}-\mathrm{Sn}, \mathrm{Bi}-\mathrm{Pb}$ and $\mathrm{Bi}^{-} \mathrm{Sn}^{-}$ $\mathrm{Pb}$ Solders, J. Japan Inst Metals, (in Japanese), Vol. 57, No. 4 (1993), p. 455-462.

(9) Takahashi, H., Mukai, M., Kawakami, T. and Takahashi, K., Crack Propagation Behavior of Sn63-Pb37 Solder, Proceedings of the JSME Spring Annual Meeting, (in Japanese), No. 97-1 (1997), p. 239-240. 